\title{
Population III Star Formation During and After the Reionization Epoch
}

\author{
Michele Trenti \\ Department of Astrophysical \& Planetary Sciences, University of Colorado, CASA, 389-UCB, \\ Boulder, CO 80309, USA
}

\begin{abstract}
.
Population III star formation during the dark ages shifted from minihalos $\left(\sim 10^{6} M_{\odot}\right)$ cooled via molecular hydrogen to more massive halos $\left(\sim 10^{8} M_{\odot}\right)$ cooled via Ly- $\alpha$ as Lyman-Werner backgrounds progressively quenched molecular hydrogen cooling. Eventually, both modes of primordial star formation were suppressed by the chemical enrichment of the IGM. We present a comprehensive model for following the modes of Population III star formation that is based on a combination of analytical calculations and cosmological simulations. We characterize the properties of the transition from metal-free star formation to the first Population II clusters for an average region of the Universe and for the progenitors of the Milky Way. Finally, we highlight the possibility of observing the explosion of Population III stars within Ly- $\alpha$ cooled halos at redshift $z \sim 6$ in future deep all sky surveys such as LSST.
\end{abstract}

Keywords: cosmology: theory, galaxies: high-redshift, Galaxy: evolution, intergalactic medium, stars: abundances, stars: formation

PACS: 97.20.Wt, 98.35.Bd, 98.62.Ai, 98.62.Bj

\section{INTRODUCTION}

The first luminous objects in the Universe, metal-free or Population III stars, are thought to form in dark matter halos with virial temperatures $T_{v i r} \gtrsim 10^{3} \mathrm{~K}$ corresponding to masses $M_{h} \sim 10^{5}-10^{6} M_{\odot}$, depending on redshift [1]. In these "minihalos", molecular hydrogen cooling lowers the gas temperature to $\sim 200 \mathrm{~K}$, which leads to the formation of either a single star or a binary that may be very massive $\left(O\left(100 M_{\odot}\right)\right)[2,3,4,5]$. The first stars begin forming in mihihalos very early in the Universe, at redshift $z \gtrsim 60$ $[6,7]$, and might provide the first seeds of supermassive black holes if their mass is sufficient to directly collapse into black holes with $M_{B H} \gtrsim 100 M_{\odot}$ [8]. Interestingly, the locations of minihalos hosting the very first stars are not strongly correlated with the most massive clusters at $z=0$. Their remnants instead likely reside in halos with masses $M_{h} \lesssim 3 \times 10^{13} M_{\odot}$ today [9].

As redshift decreases, the metal-free star formation rate rapidly increases because minihalos become progressively more abundant, until negative feedback by Lyman Werner (LW) photons photodissociates $\mathrm{H}_{2}$ molecular and increases the minimum halo mass required for cooling [10]. This happens at $z \lesssim 35$, once Population III star number densities $n_{\text {PopIII }}$ reach $\sim 1 \mathrm{Mpc}^{-3}$ (comoving). After that, a self-regulated phase for metal-free star formation sets in [11]. Eventually, the LW background becomes so strong that only halos with $T_{v i r} \gtrsim 10^{4} \mathrm{~K}$ are able to cool. This likely happens at $z \lesssim 15$, when the first metal-enriched galaxies have already been formed [12,13]. Metal-enriched 

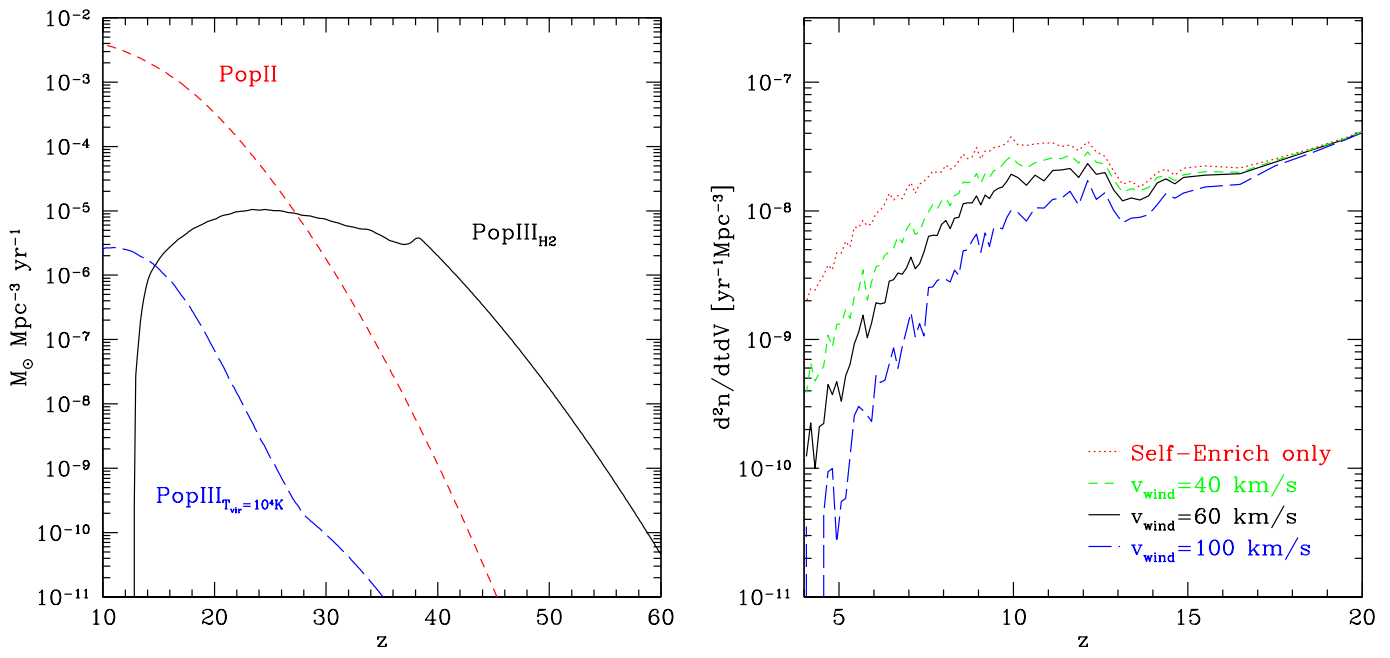

FIGURE 1. Left panel: star formation rates versus redshift predicted by our analytical model for the Population III to Population II transition (solid black line: Population III stars in minihalos; long dashed blue line: Population III stars in more massive halos, with $T_{v i r} \geq 10^{4} \mathrm{~K}$; short dashed red line: Population II stars formed out of metal-enriched gas). Figure is from [11]. Right panel: late-time Population III halo formation rate versus redshift derived from a cosmological simulation of structure formation, postprocessed to include metal enrichment by progenitor halos and by metal outflows from neighbor halos. Curves from top to bottom refer to different metal outflow velocities. Even under the assumption of efficient metal transport (high $v_{\text {wind }}$ ), pockets of metal-free gas capable of forming stars exist at $z \sim 5$. Figure is from [13].

and metal-free star formation coexist in different regions of the Universe, with metalenriched galaxies primarily in overdensities and metal-free stars in voids [13, 14]. Population III stars continue forming until $z \lesssim 5$, although at very low rates $[15,13]$. The precise details of late Population III star formation are difficult to study, however. The box sizes needed to enclose rare metal-free star formation at low redshifts, comoving volumes $\gtrsim 10^{3} \mathrm{Mpc}^{3}$, must also be able to resolve the DM halos [16, 17]. Doing this with the necesary sub-grid physics and/or post-processing analysis would severely tests the memory limits of any current model. In addition, Population III star formation might proceed differently in partially ionized gas and possibly result in less massive stars [18]. The initial mass function (IMF) of metal enriched stars is also uncertain at very high redshift because the temperature of protostellar clouds couples to the CMB temperature. Hence, a complex scenario with multiple modes of metal enriched star formation might emerge $[19,20]$.

\section{POPULATION III TO POPULATION II TRANSITION}

To address star formation in this complex framework, we start from dark-matter-only halo dynamics, based either on Press-Schechter models complemented by analytical treatments of self-enrichment [11] or on detailed halo-merger histories derived from cosmological simulations [13]. We then populate halos with stars based on an analytical 
cooling model that accounts for redshift, metallicity and radiative feedback in the LW bands (see [11] for details).

Figure 1 shows our results: metal-free star formation is an extended process over redshift. Only at extremely high redshift $(z \gtrsim 35)$ does Population III star formation depends just on local halo properties. Based on nomenclature by [21], these are Population III.1 stars. At lower redshift, metal-free star formation is also affected by radiative feedback, and such stars are Population III.2 stars. Some regions of the Universe continue to host Population III stars down to relatively low redshift $(z \lesssim 5)$, although at very low rates (right panel of Figure 1). These late-time Population III stars form in halos with $T_{v i r} \gtrsim 10^{4} \mathrm{~K}$ that are massive enough to cool even in presence of a strong LW background. The dominant process that quenches Population III star formation under these conditions is wind enrichment from neighbor protogalaxies. Because of this, late-time metal-free sources tend to be antibiased, and are preferentially formed in underdense regions [14]. Even if they are clustered, these sources are too faint for direct detection in the near future. A $100 M_{\odot}$ star at $z=6$ is about 7 magnitudes fainter than the detection limit of the James Webb Space Telescope for a deep field survey. However, a small cluster of Population III stars could be observed if magnified by gravitational lensing [14]. Alternatively, all-sky surveys reaching $M_{A B} \sim 26$ could detect Population III stars if they explode as bright supernovae [13].

Our model also highlights that metal-enriched star formation becomes the dominant mode of star formation at $z \lesssim 25$ (left panel of Figure 1). This implies that Population III stars play a minor role in the reionization of the Universe [11], with normal galaxies producing most of the ionizing photons [22].

Because direct investigation of the properties of metal-free stars is difficult, the properties of the most metal-poor stars in the local Universe have been studied to infer the IMF of their progenitors $[23,24,25,26]$. The idea behind these "galactic archeology" campaigns is that extremely metal-poor stars are likely to be objects formed out of gas enriched only by one previous generation of stars. If the first generation of stars is very massive $\left(M \gtrsim 100 M_{\odot}\right)$, then nucleosynthetic signatures of pair-instability supernovae should be found in the abundance patterns of extremely metal poor stars [27, 26]. The absence of such patterns to date has been interpreted as evidence that the Population III IMF lacks very massive stars [28].

However, this conclusion ignores wind enrichment and hence rests on the assumption that observed extremely metal poor stars $\left(Z \sim 10^{-3.5} Z_{\odot}\right)$ formed out of gas that was enriched by Population III stars. Our cosmological simulations instead show that metal outflows from dwarf galaxies enrich the majority of the extremely metal poor gas present in Milky Way progenitors (Figure 2 and [29]). This implies that galactic archeology primarily probes the IMF of Population II stars, so the absence of pair-instability signatures is not surprising. The IMF of Population III stars remains an open question for the decade to come.

\section{ACKNOWLEDGMENTS}

It is a pleasure to thank Daniel Whalen, Naoki Yoshida and Volker Bromm for the excellent organization of the meeting. MT acknowledges support from NASA 


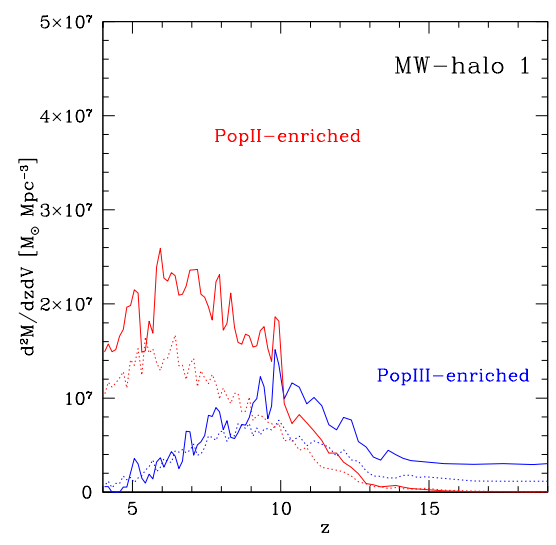

FIGURE 2. Formation rate per unit redshift of extremely metal poor gas $\left(Z \sim 10^{-3.5} Z_{\odot}\right)$ as measured from a cosmological simulations that take into account radiative feedback for Population III formation, self-enrichment of halos, and metal winds propagating at $60 \mathrm{~km} \mathrm{~s}^{-1}$ (see Trenti et al. 13). The rate of gas enriched by Pop III stars is shown as a blue lines while the rate for Pop II-enriched gas is shown as red lines. Solid lines refer to a Milky Way like halo. Dotted lines to an average region of the Universe. The majority of extremely low metallicity gas is enriched at relatively low redshift $(z \lesssim 10)$ by wind outflows. Figure from [29].

(NNX07AG77G) and NSF (AST07-07474).

\section{REFERENCES}

1. M. Tegmark, J. Silk, M. J. Rees, A. Blanchard, T. Abel, and F. Palla, ApJ 474, 1 (1997).

2. T. Abel, G. L. Bryan, and M. L. Norman, Science 295, 93 (2002).

3. V. Bromm, and R. B. Larson, ARAA 42, 79 (2004).

4. B. W. O'Shea, and M. L. Norman, ApJ 654, 66 (2007).

5. M. J. Turk, T. Abel, and B. O'Shea, Science 325, 601 (2009).

6. S. Naoz, S. Noter, and R. Barkana, MNRAS 373, L98 (2006).

7. M. Trenti, and M. Stiavelli, ApJ 667, 38 (2007).

8. A. Heger, C. L. Fryer, S. E. Woosley, N. Langer, and D. H. Hartmann, ApJ 591, 288 (2003).

9. M. Trenti, M. R. Santos, and M. Stiavelli, ApJ 687, 1 (2008).

10. Z. Haiman, M. J. Rees, and A. Loeb, ApJ 476, 458 (1997).

11. M. Trenti, and M. Stiavelli, ApJ 694, 879 (2009).

12. M. Ricotti, N. Y. Gnedin, and J. M. Shull, ApJ 685, 21 (2008).

13. M. Trenti, M. Stiavelli, and J. M. Shull, ApJ 700, 1672 (2009).

14. M. Stiavelli, and M. Trenti, ApJL 716, L190 (2010).

15. L. Tornatore, A. Ferrara, and R. Schneider, MNRAS 382, 945 (2007).

16. J. S. Bagla, and S. Ray, MNRAS 358, 1076 (2005).

17. M. Trenti, B. D. Smith, E. J. Hallman, S. W. Skillman, and J. M. Shull, ApJ 711, 1198 (2010).

18. N. Yoshida, K. Omukai, and L. Hernquist, ApJL 667, L117 (2007).

19. B. D. Smith, M. J. Turk, S. Sigurdsson, B. W. O'Shea, and M. L. Norman, ApJ 691, 441 (2009).

20. R. Schneider, and K. Omukai, MNRAS 402, 429 (2010).

21. J. C. Tan, and C. F. McKee, 2008, vol. 990 of AIP Conference Series, pp. 47.

22. M. Trenti, et al., ApJL 714, L202 (2010).

23. K. Freeman, and J. Bland-Hawthorn, ARAA 40, 487 (2002).

24. T. C. Beers, and N. Christlieb, ARAA 43, 531 (2005).

25. A. Frebel, J. L. Johnson, and V. Bromm, MNRAS 380, L40 (2007). 
26. J. Tumlinson, A. Venkatesan, and J. M. Shull, ApJ 612, 602 (2004).

27. A. Heger, and S. E. Woosley, ApJ 567, 532 (2002).

28. J. Tumlinson, ApJ 641, 1 (2006).

29. M. Trenti, and J. M. Shull, ApJ 712, 435 (2010). 